\title{
Strict Separability and Identifiability of a Class of ICA Models
}

\author{
Juan J. Murillo-Fuentes*, Member, IEEE, Rafael Boloix-Tortosa, Member, IEEE
}

\begin{abstract}
In this letter we focus on the application of independent component analysis (ICA) to a class of overdetermined blind source separation (BSS) problems. The mixing matrix in the BSS model is the product of an unknown square diagonal matrix and a projection matrix. The last matrix performs a known projection to the same or larger dimensional space. We demonstrate the conditions for the model to be strictly separable and identifiable under the statistical independence condition, paying attention to permutations and relative scalings. These results find application, e.g., in the channel estimation of ZP-OFDM and Precoded-OFDM systems.
\end{abstract}

Index Terms-EDICS: SAS-ICAB (Application to COMOFDM, COM-ESTI)

Blind source separation, overdetermined ICA, array signal processing, higher-order statistics, separability, identifiability, blind equalization, precoding, OFDM.

\section{INTRODUCTION}

Independent component analysis (ICA) [1], [2] finds application in several fields such as communications [3], [4], biomedical signal processing (electrocardiogram and electroencephalogram analysis, fMRI, brain imaging), condition monitoring, image processing [5], financial data analysis, seismic exploration, classification, or data compression and coding, among others. ICA applied to blind source separation (BSS) [6], [7] aims to estimate a set of unobservable sources from another set of observations. In the simplest case, these observations are linear instantaneous combinations of the sources and hence regarded as mixtures. The mixtures can be written as a product of the sources by a matrix, denoted as mixing matrix. If the number of mixtures equals the number of sources and the mixing matrix is of full column rank, ICA estimates this matrix by forcing independence at the output, i.e. between estimated sources. Unequal number of mixtures and sources yields to the underdetermined (less mixtures than sources) [8], [9] or the overdetermined (more mixtures than sources) [10], [11], [12] cases. In the overdetermined scenario, a prewhitening stage may be used to reduce the dimension [13].

In this letter we face ICA applied to a model of BSS where the mixing matrix has a particular known structure. The mixing matrix is the product of an unknown square diagonal matrix

Copyright (c) 2008 IEEE. Personal use of this material is permitted. However, permission to use this material for any other purposes must be obtained from the IEEE by sending a request to pubs-permissions@ieee.org.

This work was partially funded by Spanish government (Ministerio de Ciencia e Innovación TEC2009-14504-C02-02/TCM, COMONSENS) and the European Union (FEDER).

R. Boloix-Tortosa and J.J. Murillo-Fuentes are with DTSC, Escuela Superior de Ingenieros, Universidad de Sevilla, Camino de los Descubrimientos s/n, 41092 Sevilla, Spain. Tel: +34 954488150. Fax: +34 954487341. E-mail: \{rboloix, murillo\}aus.es. by a known square or overdetermined matrix. This problem can be solved by using second order (SOS) or higher order statistics (HOS). The previously described model arises, e.g., in some communication scenarios. In zero padding OFDM (ZP-OFDM) the model of the system can be simplified to a product of a diagonal matrix containing the FFT of the channel coefficients by a matrix that performs the IFFT of length $m$ of the $n$ transmitted symbols padded with zeros, $m \geq n$ [14], [15]. In cyclic prefix OFDM (CP-OFDM) we can introduce a linear precoding to later estimate the transmitted symbols, and the channel, by forcing statistical independence. In this case the system model reduces to a square matrix, the product of a diagonal matrix with the FFT of the channel coefficients by a precoding matrix. The design of this precoding matrix is an open problem [16]. In these scenarios, ICA can be exploited to achieve fast convergence and accurate results [4]. In the overdetermined ICA, e.g. in the channel estimation of ZPOFDM, the estimation of the diagonal matrix is complicated if we perform a prewhitening stage [13] and other approaches should be used [10], [11], [12].

We focus on the study of the existence of solutions to this model, either in the square or the overdetermined scenario. The definitions that characterize the solution of BSS based on the independence of the outputs are separability, identifiability and uniqueness. These features were first described by Comon in [1], later extended in [17] and other works, and recently reviewed in [18], [19]. In these works the authors propose and demonstrate the conditions for the BSS-ICA general model to have a solution, i.e., if it is possible to recover the sources, up to permutations and scaling, by imposing statistical independence at the output. They also focus on identifying the way the sources were mixed, and if the mixtures can only be generated for a given set of source distributions. We review these definitions to tailor them to the described mixing model. We aim at demonstrating if the model is (strictly) separable with no permutation or scaling. Also, we focus on the estimation or (strict) identification of the diagonal matrix, and if this estimation is unique by only imposing separation at the output. These tools are of major interest if we are to apply ICA tools to this model and, to our best knowledge, no explicit theoretical descriptions on these issues have been previously addressed.

The following material is organized as follows. We first review in Section III the BSS signal model, present a particular model and study its solution. In Section III we study the definitions of separability and identifiability and redefine them as strict separability and identifiability to endow them with new properties. In Sections [V] and $\mathrm{V}$ we demonstrate the conditions for the model to be strictly separable and 
identifiable. We end with conclusions.

\section{A CLASS OF BSS MODELS}

In its simplest form, the BSS problem accepts the following matrix model. The entries of a sensor-output vector $\mathbf{x}(t)=\left[x_{1}(t), \ldots, x_{m}(t)\right]^{\mathrm{T}}$ are instantaneous linear combinations of a set of unobservable source signals $\mathbf{s}(t)=$ $\left[s_{1}(t), \ldots, s_{n}(t)\right]^{\mathrm{T}}$ :

$$
\mathbf{x}(t)=\mathbf{A} \mathbf{s}(t),
$$

where $\mathbf{A}_{m \times n}$ represents the mixing matrix. The couple $(\mathbf{A}, \mathbf{s})$ is called a representation of r.vc. $\mathbf{x}$. It is also assumed that representations are reduced in the sense that columns in mixing matrices are not pair wise linearly dependent. If the mixing matrix $\mathbf{A}$ is of full column rank, the sources are mutually independent and at most one of them is Gaussian, it is possible [1], [17] to obtain a separation matrix $\mathbf{B}_{n \times m}$ and estimate the sources as

$$
\mathbf{y}(t)=\mathbf{B} \mathbf{x}(t)=\mathbf{B A} \mathbf{s}(t)=\mathbf{C s}(t) .
$$

However, since the scale and order of the components of $\mathbf{s}(t)$ do not affect their statistical independence, satisfactory separation is characterized by a global matrix $\mathbf{C}_{n \times n}$ with a non-mixing structure, that is, with a single non-null element per row and per column. Hence, the global matrix $\mathbf{C}$ can be written as the product of an invertible diagonal matrix and a permutation matrix.

In this paper we deal with the following mixture model:

$$
\mathbf{x}(t)=\mathbf{A} \mathbf{s}(t)=\mathbf{\Lambda} \mathbf{V} \mathbf{s}(t),
$$

where $\mathbf{V}_{m \times n}$ is a known tall matrix, $\boldsymbol{\Lambda}_{m \times m}$ is an unknown invertible diagonal square matrix and $m \geq n$. Notice that this model is not a decomposition of the one in (11), it is just a subset within the general linear instantaneous ICA model. We can apply well-known ICA algorithms to estimate the sources by using the model in (3). However, we do not exploit the knowledge on the structure of the mixing. We may better solve the mixture by adopting the following decomposition for the separating matrix,

$$
\mathbf{y}(t)=\mathbf{B A} \mathbf{s}(t)=\mathbf{V}^{\dagger} \mathbf{D} \mathbf{\Lambda} \mathbf{V} \mathbf{s}(t)=\mathbf{C s}(t),
$$

where $(\cdot)^{\dagger}$ is the Moore-Penrose inverse and $\mathbf{D}$ is a diagonal matrix that yields separation. In the general model in (1) we have to estimate $n \times m$ entries, up to scaling and permutations, to invert the effect of the mixing matrix. In the model in (3) we just need to estimate the diagonal matrix. While in the general model we must use higher order statistics (HOS), the model in (3) may be usually solved by using second order statistics (SOS). By forcing decorrelation at the output we have $n(n+1) / 2$ different equations of the unknown $m$ entries of the unknown diagonal matrix. If we then apply HOS, we can further improve the solution.

\section{SeParability AND IDENTIFIABILITy}

In the remaining of this paper we focus on the separability and indentifiability of the model in (3). Separability and indentifiability of the general model in (1) have been recently reviewed in [18]. The model in (1) is separable "if for every matrix $\mathbf{B}$ such that $\mathbf{B x}(t)$ has $n$ independent components, we have $\mathbf{B x}(t)=\mathbf{C s}(t)$ for some global matrix $\mathbf{C}$ with nonmixing structure. Moreover, matrix B must exist". A mixture as in (1) is separable if the mixing matrix is of full column rank and at most one source variable is normal [1]. The model is identifiable "if in every reduced representations $(\mathbf{A}, \mathbf{s})$ and $(\mathbf{E}, \mathbf{r})$ of $\mathbf{x}$, every column of $\mathbf{A}$ is linearly dependent on some column of $\mathbf{E}$ and vice versa". Separability implies identifiability: if we have two mixing matrices yielding the same mixtures, every column of one of the matrices is linearly dependent on some column of the second and vice versa. Hence, if we have two separating matrices, every row of one matrix is linearly dependent on some row of the second.

The model in (3) has some special and interesting features compared to the general BSS model. We next define two new characteristics. Later in this letter, we demonstrate the conditions for the model to achieve them.

Definition 1: (Strictly separable) if for any $\mathbf{B x}(t)=\mathbf{C s}(t)$ with independent components, where $\mathbf{B}=\mathbf{V}^{\dagger} \mathbf{D}$ as in (4), we have $\mathbf{C}=a \mathbf{I}$, for any non-null constant $a$, i.e., the separation is achieved up to a common scale factor with no permutation.

Definition 2: (Strictly identifiable via separation) if for any $\mathbf{B x}(t)=\mathbf{C s}(t)$ with independent components, we have $\mathbf{D}=$ $a \boldsymbol{\Lambda}^{-1}$ for any non-null constant $a$, where $\mathbf{B}=\mathbf{V}^{\dagger} \mathbf{D}$ and $\boldsymbol{\Lambda}$ as in (4), i.e., the separation yields the estimation of the unknown mixing diagonal matrix up to a constant.

We define strictly identifiable via separation, to emphasize we focus on the estimation of the mixture by only separating the sources. Notice that unlike in the separable/identifiable definitions [18], and as we demonstrate later in this paper, strict separability does not imply strict identifiability via separation. We do not focus on estimating the mixing matrix A, but the diagonal matrix $\boldsymbol{\Lambda}$. In order to give more intuition to these definitions, consider the following example.

Example 1: As a example of a model that it is separable but it is not strictly separable we present the following,

$$
\mathbf{V}=\left(\begin{array}{lll}
1 & 0 & 0 \\
0 & 1 & 0 \\
0 & 0 & 1 \\
1 & 0 & 0
\end{array}\right)
$$

and any diagonal matrix $\Lambda$ so that the mixing matrix $\Lambda \mathrm{V}$ is of full column rank, and therefore separable. Suppose $\boldsymbol{\Lambda}=$ $\operatorname{diag}([\alpha, \beta, \gamma, \delta])$ where $\alpha, \beta, \gamma$ and $\delta$ are any constants. Then we left multiply the mixture by $\mathbf{V}^{\dagger}$. At the output we have the sources separated as $\mathbf{V}^{\dagger} \boldsymbol{\Lambda} \mathbf{V} \mathbf{s}(t)=\operatorname{diag}((\alpha+\delta) / 2, \beta, \gamma) \mathbf{s}(t)$, where we can independently scale every source. Hence, it is not strictly separable.

Suppose we have two separating diagonal matrices, one given by the diagonal matrix $\mathbf{D}_{1}$ and the other by $\mathbf{D}_{2}$, by imposing the separability condition [1] it follows that

$$
\mathbf{V}^{\dagger} \mathbf{D}_{1} \Lambda \mathbf{V}=\mathbf{V}^{\dagger} \mathbf{D}_{2} \Lambda \text { VPS }
$$


where $\mathbf{P}$ and $\mathbf{S}$ are some permutation and scaling matrix, respectively. We next study this equality to show the conditions for the model to be strictly separable. Then we demonstrate the conditions for the model to be strictly identifiable via separation, i.e., that only one diagonal matrix $\mathbf{D}$ exists such that separates the sources as $\mathbf{V}^{\dagger} \mathbf{D} \mathbf{\Lambda} \mathbf{V}=a \mathbf{I}$ for some constant $a$. These properties are of great importance, only a global constant remains undetermined after imposing independency at the output, and the mixing matrix can be estimated by separation.

\section{STRICT SEPARABILITY}

For the model in (3)-(4) to be strictly separable we analyze if for some diagonal matrix, $\boldsymbol{\Sigma}$, a solution $\mathbf{D}^{\prime}=\boldsymbol{\Sigma} \mathbf{D}$, where $\mathbf{D}=\boldsymbol{\Lambda}^{-1}$, yields a permuted and scaled version of the ouputs. We have to demonstrate that the product $\mathbf{V}^{\dagger} \boldsymbol{\Sigma} \mathbf{D} \boldsymbol{\Lambda} \mathbf{V} \neq \mathbf{P S}$, where PS $\neq a \mathbf{I}$, being $a$ any global scale factor. This reduces to show that $\mathbf{V}^{\mathrm{T}} \mathbf{\Sigma} \mathbf{V} \neq \mathbf{V}^{\mathrm{T}} \mathbf{V P S}$. We next study permutation and scaling separately to reach the conditions for strict separability.

Lemma 1: (Permutation) Given a tall matrix $\mathbf{V}_{m \times n}$ of full column rank, there does not exist a diagonal matrix $\boldsymbol{\Sigma}_{m \times m}$ such that:

$$
\mathbf{V}^{\mathrm{T}} \boldsymbol{\Sigma} \mathbf{V}=\mathbf{V}^{\mathrm{T}} \mathbf{V P}
$$

for any permutation matrix $\mathbf{P}_{n \times n}$.

Proof: The product $\mathbf{V}^{\mathrm{T}} \mathbf{V}$ is a full rank symmetric matrix. It has a singular value decomposition $\mathbf{V}^{\mathrm{T}} \mathbf{V}=\mathbf{Q}^{\mathrm{T}} \mathbf{T Q}$ with non null eigenvalues. If we multiply this product by a permutation matrix $\mathbf{P}$, we have $\mathbf{V}^{\mathrm{T}} \mathbf{V P}=\mathbf{Q}^{\mathrm{T}} \mathbf{T} \mathbf{P P}$. Since the product $\mathbf{Q P}$ is unitary, if $\mathbf{P} \neq \mathbf{I}$ we have the singular value decomposition of a non-symmetric matrix, but $\mathbf{V}^{\mathrm{T}} \boldsymbol{\Sigma} \mathbf{V}$ is symmetric. Therefore, no permutation is allowed.

By this lemma we show that under the only conditions of statistical independence at the output and full column rank, the ordering of the output signals can be uniquely determined. We next face the change of scale of the sources. We first introduce the next definition.

Definition 3: (Row linked) A matrix $\mathbf{V}_{m \times n}$ is row linked if it exists a set of non-null entries, denoted as link set, such that we can travel from the $i$ th row to the $j$ th row by jumping from an element of the link set to another in the same row or the same column, for any pair $i, j=1, \ldots, m$.

Notice that if a matrix is full column rank and row linked it is column linked, we may say it is linked. Also, it is interesting to remark that the adjacent elements of one element in the link set may be null elements. The property of column linking forces the observations to be representative mixtures of all sources: we cannot arrange the observations in groups where none of the mixed sources in one group are mixed with the others. In Example 1, rows 1 an 4 are linked, but rows 2 and 3 are not. In the matrix in (12), later in this letter, all rows are connected. We can link rows number 3 and 4 through rows 2 and 1 .

Lemma 2: (Scaling) Given a row linked matrix $\mathbf{V}^{\mathrm{T}} \mathbf{V}$, where $\mathbf{V}$ is a $m \times n$ matrix of full column rank, there do not exist diagonal matrices $\boldsymbol{\Sigma}_{m \times m}$ and $\mathbf{S}_{n \times n} \neq a \cdot \mathbf{I}$, where $a$ is any constant, such that

$$
\mathbf{V}^{\mathrm{T}} \boldsymbol{\Sigma} \mathbf{V}=\mathbf{V}^{\mathrm{T}} \mathbf{V S}
$$

Proof: Assume that $\boldsymbol{\Sigma}$ and $\mathbf{S}$ exist. These matrices must fulfill (8), i.e.,

$$
\sum_{r}(\mathbf{V})_{r, k}(\mathbf{V})_{r, l}-\sum_{r}(\mathbf{V})_{r, k}(\mathbf{V})_{r, l}(\boldsymbol{\Sigma})_{r, r}(\mathbf{S})_{l, l}=0
$$

where $(\mathbf{A})_{r, q}$ denotes the entry $r, q$ of matrix A. For elements $\left(\mathbf{V}^{\mathrm{T}} \mathbf{V}\right)_{k, l}$ in the same column $l$,

$$
(\mathbf{S})_{l, l}=\frac{\sum_{r}(\mathbf{V})_{r, k}(\mathbf{V})_{r, l}}{\sum_{r}(\mathbf{V})_{r, k}(\mathbf{V})_{r, l}(\boldsymbol{\Sigma})_{r, r}}, \forall k
$$

Since matrix $\mathbf{V}^{\mathrm{T}} \mathbf{V}$ is symmetric, for some pair $k, l$ in the link set,

$$
(\mathbf{S})_{k, k}=\frac{\sum_{r}(\mathbf{V})_{r, l}(\mathbf{V})_{r, k}}{\sum_{r}(\mathbf{V})_{r, l}(\mathbf{V})_{r, k}(\mathbf{\Sigma})_{r, r}}=(\mathbf{S})_{l, l} .
$$

Equation 10 is defined for all the diagonal entries and, provided that $\mathbf{V}^{\mathrm{T}} \mathbf{V}$ is row linked, we have them all related by (11). Therefore $(\mathbf{S})_{k, k}=a$ for $k=1, \ldots, m$.

Notice that a null entry in the diagonal mixing matrix $\boldsymbol{\Lambda}$ means a null observation. Hence, with no loss of generality, we have assumed in the model that the entries of matrix $\Lambda$ are non-null.

Theorem 1: (Strict separability) Given a matrix $\boldsymbol{\Lambda}_{m \times m}$ with non-null diagonal elements, and a tall matrix $\mathbf{V}_{m \times n}$ of full column rank such that the product $\mathbf{V}^{\mathrm{T}} \mathbf{V}$ is row linked, the model in (3)-(4) is strictly separable.

Proof: The proof is immediate by Lemma 1 and Lemma 2.

The following example is included to illustrate the results on the conditions for strictly separability.

Example 2: We propose an example where the model is strictly separable but it is not strictly identifiable. Matrix $\mathbf{V}$ in (3) is

$$
\mathbf{V}=\left(\begin{array}{lll}
1 & 1 & 0 \\
0 & 1 & 1 \\
0 & 0 & 1 \\
1 & 1 & 0
\end{array}\right)
$$

and any diagonal matrix $\Lambda$ so that the mixing matrix $\Lambda \mathbf{V}$ is of full column rank, and therefore separable. Suppose we separate the sources with any matrix $\boldsymbol{\Sigma}$ such that $\boldsymbol{\Sigma} \boldsymbol{\Lambda}=$ $\operatorname{diag}([\alpha, \beta, \gamma, \delta])$ where $\alpha, \beta, \gamma$ and $\delta$ are any constants. Then we left multiply the mixture by $\mathbf{V}^{\dagger}$. At the output we have separation only if $\beta=\gamma$ and $\alpha+\delta=2 \beta$. In this case the separated sources are $\beta \mathbf{s}(t)$. Notice that we have different values of $\alpha$ and $\delta$ yielding separation, hence the model is not strictly identifiable via separation.

\section{Strict identifiability}

In the following theorem we describe the conditions for strict identifiability by imposing separation. We will use the Khatri-Rao product $\odot$ : the $k$-th column of $(\mathbf{B} \odot \mathbf{A})$ is the Kronecker product of the $k$-th column of $\mathbf{B}$ by the $k$-th column of $\mathbf{A}$.

Theorem 2: (Strict identifiability via separation) Given a diagonal matrix $\boldsymbol{\Lambda}_{m \times m}$ with non-null entries, a tall full 
column rank matrix $\mathbf{V}_{m \times n}$ and its Moore-Penrose inverse $\mathbf{V}^{\dagger}$, then there exists only one diagonal matrix $\mathbf{D}=\boldsymbol{\Lambda}^{-1}$ such that

$$
\mathbf{V}^{\dagger} \mathbf{D} \boldsymbol{\Lambda} \mathbf{V}=\mathbf{I}
$$

iff the matrix $\tilde{\mathbf{U}}^{\mathrm{T}} \odot \tilde{\mathbf{U}}^{\mathrm{T}}$ has rank equal to $m$, where $\tilde{\mathbf{U}}$ is the left matrix in the singular-value decomposition (SVD) of matrix $\mathbf{V}$,

$$
\mathbf{V}=\mathbf{U} \boldsymbol{\Delta} \mathbf{W}^{\mathrm{T}}=\mathbf{U}\left[\begin{array}{c}
\tilde{\boldsymbol{\Delta}} \\
0
\end{array}\right] \mathbf{W}^{\mathrm{T}}=\tilde{\mathbf{U}} \tilde{\boldsymbol{\Delta}} \mathbf{W}^{\mathrm{T}} .
$$

Proof: It follows that if

$$
\mathbf{V}^{\dagger} \mathbf{D} \boldsymbol{\Lambda} \mathbf{V}=\mathbf{W} \tilde{\boldsymbol{\Delta}}^{-1} \tilde{\mathbf{U}}^{\mathrm{T}} \mathbf{D} \boldsymbol{\Lambda} \tilde{\mathbf{U}} \tilde{\boldsymbol{\Delta}} \mathbf{W}^{\mathrm{T}}=\mathbf{I},
$$

then $\tilde{\mathbf{U}}^{\mathrm{T}} \mathbf{D} \boldsymbol{\Lambda} \tilde{\mathbf{U}}=\mathbf{I}_{n \times n}$. Denoting the product $\mathbf{D} \boldsymbol{\Lambda}$ by $\mathbf{K}$, equation (13) holds iff

$$
\sum_{l}(\tilde{\mathbf{U}})_{l, i}(\mathbf{K})_{l, l}(\tilde{\mathbf{U}})_{l, j}=\delta_{i, j}
$$

where $\delta_{i, j}=1$ if $i=j$ and 0 if $i \neq j$. This can be written in matrix form as follows

$$
\operatorname{vec}\left\{\tilde{\mathbf{U}}^{\mathrm{T}} \mathbf{K} \tilde{\mathbf{U}}\right\}=\left(\tilde{\mathbf{U}}^{\mathrm{T}} \odot \tilde{\mathbf{U}}^{\mathrm{T}}\right) \operatorname{vecd}\{\mathbf{K}\}=\operatorname{vec}\{\mathbf{I}\},
$$

where the operator $\operatorname{vec}\{\cdot\}$ indicates a stacking of the columns of a matrix into a vector and the operator vecd $\{\cdot\}$ is a column vector with the diagonal values. Hence, it follows that only when the rank of the system is equal to $m$ we have a unique diagonal matrix inverting the mixture.

The system in (17) has $n^{2}$ equations. Any system with $n^{2}<$ $m$ is not strictly identifiable. In Example 2 we need $m=4$ linearly independent equations in (17) and we have only 3 . Another example of non strict identifiability is a matrix $\mathbf{V}$ with one or more null rows. In this case $\tilde{\mathbf{U}}^{\mathrm{T}}=\left[\overline{\mathbf{U}}_{p \times p} \mathbf{0}\right]$, for some $p$, and we have infinite solutions.

The immediate solution to (4) with no permutation or scaling of the outputs is $\mathbf{B}=\mathbf{V}^{\dagger} \boldsymbol{\Lambda}^{-1}$, it exists and is unique as stated in Theorem 2. By Lemma 1 we show that, under mild conditions, the outputs cannot be permuted, a different D cannot lead to the same solution with the outputs permuted. And by Lemma 2 we demonstrate the conditions under which the outputs cannot be independently scaled, no other solution exists with different scalings.

\section{CONCLUSIONS}

In this letter we have focused in a particular problem of array processsing. We propose this multiple input multiple output model to be solved by using ICA, i.e., by imposing statistical independence at the output. The ICA tools based either in SOS or HOS can be applied. We study if the standard definitions of separability and identifiability can be further extended, provided the complexity of the problem is reduced compared to the general ICA model. We prove that these features can be redefined to make them more restrictive, and we do demonstrate the conditions on the model to achieve them. These results are useful when applying ICA to this model, e.g., in the channel estimation of ZP-OFDM and linearly precoded OFDM systems. In the ZP-OFDM we can study if the sources can be recovered up to a global scaling when applying ICA to estimate the channel. In the precoded OFDM we provide the conditions for the precoding matrix to be useful, from the separation point of view.

\section{REFERENCES}

[1] P. Comon, "Independent component analysis, a new concept?" Signal Processing, vol. 36, no. 3, pp. 287-314, Apr 1994.

[2] A. Hyvärinen, J. Karhunen, and E. Oja, Independent component analysis. John Willey and Sons, 2001.

[3] A. Caamaño-Fernández, R. Boloix-Tortosa, J. Ramos, and J. J. MurilloFuentes, "Hybrid higher-order statistics learning in multiuser detection," IEEE Trans. on Man and Cybernetics Part C., vol. 34, no. 4, pp. 417424, Nov 2004.

[4] R. Boloix-Tortosa and J. J. Murillo-Fuentes, "Blind source separation in the adaptive reduction of inter-channel interference for OFDM," Lecture Notes on Computer Sciences, vol. 3195, pp. 1142-1149, 2004.

[5] J. J. Murillo-Fuentes, "Independent component analysis in the blind watermarking of digital images," Neurocomputing, vol. 70, pp. 28812890, 2007.

[6] J. F. Cardoso, "Blind signal separation: Statistical principles," Proc. of the IEEE, vol. 86, no. 10, pp. 2009-2025, Oct 1998.

[7] S. Haykin, Ed., Unsupervised Adaptive Learning. Volume I Blind Source Separation. NY: Wiley Interscience, 2000, ch. Blind Signal Separation and Extraction: Neural and Information-Theoretic Approaches, pp. 63138.

[8] M. S. Lewicki and T. J. Sejnowski, "Learning overcomplete representations," Neural Computation, vol. 12, no. 2, pp. 337-365, 2000.

[9] T. Lee, M. Lewicki, M. Girolami, and T. Sejnowski, "Blind source separation of more sources than mixtures using overcomplete representations," IEEE Signal Proc. Lett., vol. 6, no. 4, pp. 87-90, 1999.

[10] J. V. Stone and J. Porrill, "Undercomplete independent component analysis for signal separation and dimension reduction," Technical report, 1998.

[11] L. Zhang, A. Cichocki, and S. Amari, "Natural gradient algorithm for blind separation of overdetermined mixture with additive noise," IEEE Signal Processing Lett., vol. 6, no. 11, pp. 293-295, Nov. 1999.

[12] S. Squartini, F. Piazza, and F. J. Theis, "New riemannian metrics for speeding-up the convergence of over- and underdetermined ICA." in Proc. ISCAS, 2006.

[13] M. Joho, H. Mathis, and R. Lambert, "Overdetermined blind source separation: Using more sensors than source signals in a noisy mixture," in Proc. ICA, Helsinki, Finland, Jun 2000, pp. 81-86.

[14] B. Muquet, Z. Wang, G. B. Giannakis, M. de Courville, and P. Duhamel, "Cyclic prefixing or zero padding for wireless multicarrier transmissions?" IEEE Trans. on Communications, vol. 50, no. 12, pp. 21362148, December 2002.

[15] R. Boloix-Tortosa, F. Payan-Somet, and J. Murillo-Fuentes, "Reduced complexity blind equalization schemes for ZP-OFDM systems," in in Proc. IEEE SPAWC 2007, June 2007, pp. 1-5.

[16] F. Gao and A. Nallanathan, "Blind channel estimation for OFDM systems via a generalized precoding," IEEE Trans. Vehicular Technology, vol. 56, no. 3, pp. 1155-1164, May 2007.

[17] X. R. Cao and R. W. Liu, "General approach to blind source separation," IEEE Trans. on Signal Processing, vol. 44, no. 3, pp. 562-571, 1996.

[18] J. Eriksson and V. Koivunen, "Identifiability, separability and uniqueness of linear ICA models," IEEE Signal Processing Lett., vol. 11, no. 7, pp. 601-604, July 2004.

[19] — - "Complex random vectors and ica models: identifiability, uniqueness, and separability," Information Theory, IEEE Transactions on, vol. 52, no. 3, pp. 1017-1029, March 2006. 